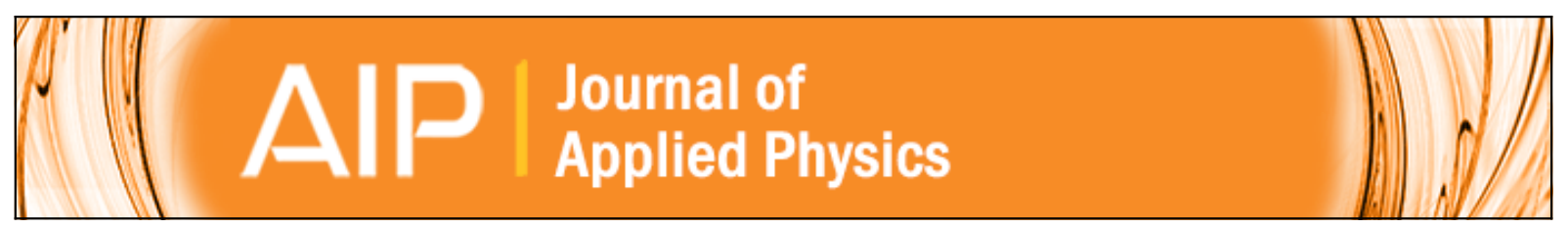

\title{
Monolayers of MoS2 as an oxidation protective nanocoating material
}

H. Sener Sen, H. Sahin, F. M. Peeters, and E. Durgun

Citation: Journal of Applied Physics 116, 083508 (2014); doi: 10.1063/1.4893790

View online: http://dx.doi.org/10.1063/1.4893790

View Table of Contents: http://scitation.aip.org/content/aip/journal/jap/116/8?ver=pdfcov

Published by the AIP Publishing

\section{Articles you may be interested in}

Surface oxidation energetics and kinetics on MoS2 monolayer

J. Appl. Phys. 117, 135301 (2015); 10.1063/1.4916536

Optimal electron irradiation as a tool for functionalization of MoS2: Theoretical and experimental investigation

J. Appl. Phys. 117, 135701 (2015); 10.1063/1.4916530

Joint first-principles/continuum calculations of electromechanical properties of MoS2 monolayer

Appl. Phys. Lett. 105, 061910 (2014); 10.1063/1.4893360

Atomistic simulation of the electronic states of adatoms in monolayer MoS2

Appl. Phys. Lett. 104, 141603 (2014); 10.1063/1.4870767

The pristine atomic structure of MoS2 monolayer protected from electron radiation damage by graphene Appl. Phys. Lett. 103, 203107 (2013); 10.1063/1.4830036

Frustrated by

old technology?

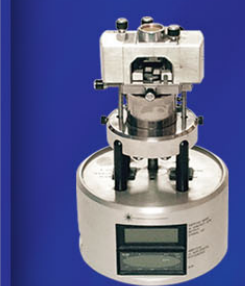

Is your AFM dead

and can't be repaired?

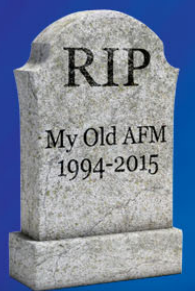

Sick of bad customer support?

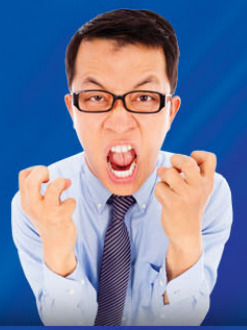

It is time to upgrade your AFM Minimum $\$ 20,000$ trade-in discount for purchases before August 31st

Asylum Research is today's technology leader in AFM

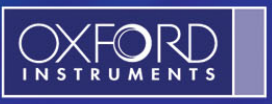




\title{
Monolayers of $\operatorname{MoS}_{2}$ as an oxidation protective nanocoating material
}

\author{
H. Sener Sen, ${ }^{1}$ H. Sahin, ${ }^{2}$ F. M. Peeters,${ }^{2}$ and E. Durgun ${ }^{1,3, a)}$ \\ ${ }^{1}$ UNAM-National Nanotechnology Research Center, Bilkent University, Ankara 06800, Turkey \\ ${ }^{2}$ Department of Physics, University of Antwerp, 2610 Antwerp, Belgium \\ ${ }^{3}$ Institute of Materials Science and Nanotechnology, Bilkent University, Ankara 06800, Turkey
}

(Received 3 July 2014; accepted 12 August 2014; published online 26 August 2014)

\begin{abstract}
First-principle calculations are employed to investigate the interaction of oxygen with ideal and defective $\mathrm{MoS}_{2}$ monolayers. Our calculations show that while oxygen atoms are strongly bound on top of sulfur atoms, the oxygen molecule only weakly interacts with the surface. The penetration of oxygen atoms and molecules through a defect-free $\mathrm{MoS}_{2}$ monolayer is prevented by a very high diffusion barrier indicating that $\mathrm{MoS}_{2}$ can serve as a protective layer for oxidation. The analysis is extended to $\mathrm{WS}_{2}$ and similar coating characteristics are obtained. Our calculations indicate that ideal and continuous $\mathrm{MoS}_{2}$ and $\mathrm{WS}_{2}$ monolayers can improve the oxidation and corrosionresistance of the covered surface and can be considered as an efficient nanocoating material. (C) 2014 Author(s). All article content, except where otherwise noted, is licensed under a Creative Commons Attribution 3.0 Unported License. [http://dx.doi.org/10.1063/1.4893790]
\end{abstract}

\section{INTRODUCTION}

Depending on the requirements on the functionality of an application, such as, reduction of friction forces (lubrication), passivation of chemical reactivity, and/or protection from corrosion/wear, surface coating has always been an active research area in different fields. At macroscale, a surface can be covered by different materials including paints, ${ }^{1}$ polymers, ${ }^{2}$ organic layers, ${ }^{3-5}$ metals, and alloys. ${ }^{6}$ Conventional coating materials modify the structural and physical properties of the underlying structure which can result in undesired alterations. These effects are more drastic in reduced dimensions, especially in nanoscale systems. Therefore, it is essential to find a suitable material that protects the surface without losing the desired properties. With this motivation, theoretical and experimental research on novel coating materials of a few atomic layer thickness have emerged. Being an ultra-thin, strong and light material, graphene ${ }^{7}$ has been viewed as an ideal nanocoating material. Various metal surfaces including $\mathrm{Ni},{ }^{8,9} \mathrm{Ru}(0001),{ }^{10} \mathrm{Cu} / \mathrm{Ni}$ alloy, ${ }^{11} \mathrm{Cu},{ }^{12,13} \operatorname{Ir}(111)$, and $\operatorname{Pt}(111)^{14}$ have been coated by graphene and a reduction in the oxidation of the surface was reported. It was theoretically shown that even graphene itself strongly interacts with oxygen atoms, it poses a high energy barrier for the penetration of oxygen and thus can protect the surface underneath against oxidation as long as the graphene coating is defect free. ${ }^{15}$

Recent advances made growth and exfoliation of single layers of lamellar materials beyond graphene also possible. Among these novel materials, two-dimensional $\mathrm{MoS}_{2}{ }^{16}$ which belongs to the family of transition metal dichalcogenides (TMD), has been of special interest for nanocoating since its bulk form is a well-known coating material at macro scale. ${ }^{17-19} \mathrm{MoS}_{2}$ crystals are composed of vertically stacked layers with an interlayer distance of $6.5 \AA$ (JCPDS 77-1716) interacting via Van der Waals ( $\mathrm{vdW}$ ) forces, similar to graphite. ${ }^{20}$ The unit cell of $\mathrm{MoS}_{2}$ consists of a Mo-layer sandwiched between two S-layers. Each of these sub-layers

\footnotetext{
${ }^{a)}$ Electronic mail: durgun@unam.bilkent.edu.tr
}

has a hexagonal structure in plane and $\mathrm{S}$ atoms are chemically bonded with the Mo atoms in a trigonal prismatic fashion. Weak interatomic interactions between its layered structures allow easy and low-strength shearing. $\mathrm{MoS}_{2}$ can be used as solid lubricant when load carrying capacity, operating temperature, and friction are crucial parameters and liquid lubricants are impractical. ${ }^{17-19}$ It is also shown that thin films of fullerene-like $\mathrm{MoS}_{2}$ nanoparticles have an ultralow friction coefficient in ambient conditions, which makes them an ideal material for tribological applications. ${ }^{17}$ In the ultimate limit of a single layer, $\mathrm{MoS}_{2}$ posses different optical, mechanical, and electrical properties than its bulk phase. Bulk $\mathrm{MoS}_{2}$, for instance, is a semiconductor with an indirect band gap of $1.3 \mathrm{eV}$, whereas monolayer $\mathrm{MoS}_{2}$ is a direct band gap semiconductor with a band gap of $1.8 \mathrm{eV} .^{21-23}$ New emerging properties allow single or a few-layered $\mathrm{MoS}_{2}$ to be used in different fields such as photocatalyst, ${ }^{24,25}$ a field effect transistor, ${ }^{26,27}$ and photosensitive thin film for solar applications. ${ }^{28,29}$ Although oxygen adsorption on $\mathrm{MoS}_{2}$ monolayers ${ }^{30,31}$ and effects of oxygen on device applications have been examined, ${ }^{27}$ the possibility of using $\mathrm{MoS}_{2}$ monolayers as a protective coating material against oxidation for reactive surfaces has not been considered yet.

In this work, we study the interaction of oxygen (adsorption and diffusion) with a $\mathrm{MoS}_{2}$ monolayer for potential usage in nanocoating applications. First, we examine the adsorption of oxygen atom/molecule on $\mathrm{MoS}_{2}$. Next, we determine the minimum energy path and the reaction barrier for lateral and vertical diffusion of oxygen through ideal and suspended $\mathrm{MoS}_{2}$. In addition, the possible effect of the underlaying surface is taken into account by fixing the bottom S-layer. We repeat the analysis for defective $\mathrm{MoS}_{2}$ containing various types of vacancies. Finally, the study is extended to similar structures made of monolayer $\mathrm{WS}_{2}$.

\section{METHODOLOGY}

In this study, we performed first-principles, spin-polarized calculations within density functional theory ${ }^{32,33}$ using 
the Vienna $a b$ initio simulation package (VASP). ${ }^{34,35}$ Exchange-correlation energy was expressed by the generalized gradient approximation ${ }^{36}$ including $\mathrm{vdW}$ correction and the projector augmented wave (PAW) potential ${ }^{37}$ is used with kinetic energy cutoff of $500 \mathrm{eV}$. In order to minimize the interaction of adsorbed atoms/molecules with their replica in the neighboring cells, the calculations were carried out in a $4 \times 4$ supercell with $15 \AA$ vacuum spacing in the vertical direction. In the self-consistent potential and total energy calculations, the Brillouin zone of the supercell was sampled in the $\mathbf{k}$-space using $5 \times 5 \times 1$ mesh points. ${ }^{38}$ All structures were relaxed using the Kosugi algorithm ${ }^{39}$ with simultaneous minimization of the total energy and the interatomic forces. The convergence for the total energy was set to $10^{-5} \mathrm{eV}$, and the maximum residual force allowed on each atom was fixed at $10^{-2} \mathrm{eV} / \AA$.

The energetics of oxygen vertical diffusion were calculated by forcing it to penetrate through the $\mathrm{MoS}_{2}$ layer. The minimum energy path was determined by $0.2 \AA$ vertical displacement of $\mathrm{O} / \mathrm{O}_{2}$. At each step, the lateral coordinates of oxygen were relaxed while the perpendicular coordinate was kept fixed. $\mathrm{MoS}_{2}$ is considered to be free-standing where all atoms were fully relaxed except specific Mo atoms which were kept fixed to prevent the displacement of the suspended layer. When necessary, the energy barrier of the reaction paths was calculated by using the nudged-elastic band approach. ${ }^{40}$

\section{OXYGEN ADSORPTION ON MOS ${ }_{2}$ MONOLAYER}

In order to understand the interaction of oxygen with $\mathrm{MoS}_{2}$, we start with the adsorption of a single oxygen atom on the $\mathrm{MoS}_{2}$ surface. We consider a $4 \times 4$ supercell to avoid artificial O-O interaction and consider three possible adsorption sites, namely hollow $(\mathrm{H})$, bridge (B), and top (T) as shown in Fig. 1(a). Our results indicate that independent of the initial configuration the $\mathrm{O}$ atom always prefers the top site and binds to a sulfur atom $\left(\mathrm{S}_{t}\right)$ on top (Figs. 1(a) and $1(\mathrm{c}))$. The bond length $\left(\mathrm{d}_{S-O}\right)$ is calculated as $1.48 \AA$ indicating a strong interaction between $\mathrm{O}$ and $\mathrm{S}$ atoms. The ground state of the system is non-magnetic with zero total magnetic moment. The adsorption energy $\left(\mathrm{E}_{b}\right)$ of $\mathrm{O}$ atom (and also $\mathrm{O}_{2}$ molecule) on $\mathrm{MoS}_{2}$ is given by

$$
E_{b}=E_{T}\left(M o S_{2}\right)+E_{T}\left(O_{x}\right)-E_{T}\left(M o S_{2}+O_{x}\right),
$$

where $\mathrm{E}_{\mathrm{T}}\left(\mathrm{MoS}_{2}\right), \mathrm{E}_{\mathrm{T}}\left(\mathrm{O}_{x}\right)$, and $\mathrm{E}_{\mathrm{T}}\left(\mathrm{MoS}_{2}+\mathrm{O}_{x}\right)$ are the total energy of fully optimized bare $\mathrm{MoS}_{2}(4 \times 4$ supercell), oxygen atom $(\mathrm{x}=1)$ or molecule $(\mathrm{x}=2)$, and $\mathrm{MoS}_{2}-\mathrm{O}_{x}$ system, respectively. All energies are calculated within the same supercell for the sake of comparison. Using Eq. (1), $\mathrm{E}_{b}$ of a single $\mathrm{O}$ atom is obtained as $3.93 \mathrm{eV}$ indicating a strong covalent character. The inclusion of vdW correction in our calculations does not make any significant changes without modifying the $\mathrm{d}_{S-O}$ but it only slightly increases $\mathrm{E}_{b}$ by $20 \mathrm{meV}$. Fig. 2(b) displays the total and projected density of states before and after $\mathrm{O}$ atom adsorption. This analysis shows that $2 p$ orbitals of $\mathrm{O}$ mix with $3 p$ orbitals of $\mathrm{S}$ to form a strong bond. Upon adsorption, initially three-coordinated $\mathrm{S}_{t}$ atom becomes four-coordinated, which is not unexpected as sulfur can have four-bonds in a tetragonal manner in several compounds including $\mathrm{ZnS}$ and $\mathrm{H}_{2} \mathrm{SO}_{4}$. The difference in charge density $\left[\rho\left(\mathrm{MoS}_{2}+\mathrm{O}\right)-\rho\left(\mathrm{MoS}_{2}\right)+\rho(\mathrm{O})\right]$ indicates that adsorption locally affects the electron distribution (Fig. 2(a)). The $\mathrm{O}$ atom takes only the charge from $\mathrm{S}_{t}$ but all the other atoms are not significantly affected. We quantify the charge exchange by using a Bader analysis. ${ }^{41}$ When ideal $\mathrm{MoS}_{2}$ is analyzed, the net charge on Mo and $\mathrm{S}$ atoms is $+1.05 \mathrm{e}$ and $-0.53 \mathrm{e}$, respectively. As a consequence of the adsorption, $\mathrm{S}_{t}$ donates electrons to $\mathrm{O}$ and becomes positively charged. The net charge on $\mathrm{O}$ and $\mathrm{S}_{t}$ becomes $-1.15 \mathrm{e}$ and +0.60 e, respectively. The charge exchange is of the same order as the electronegativities $(\chi)$ of the considered atoms where $\chi_{O}>\chi_{S}>\chi_{M o}$. The net charge on all the other atoms remains the same confirming the localized affect obtained by the difference charge density analysis. Accordingly, additional $\mathrm{O}$ atom(s) can bind to a neighboring $\mathrm{S}$ atom(s) in a similar manner. For instance, we try out a second $\mathrm{O}$ atom that binds to the $\mathrm{S}$ atom adjacent to $\mathrm{S}_{t}$ on top with a slightly lower $\mathrm{E}_{b}=3.52 \mathrm{eV}$. Our calculations also indicate that the substitution of $\mathrm{S}$ with $\mathrm{O}$ atoms is an endothermic process and requires high energy. The reaction barrier is calculated as $4.5 \mathrm{eV}$ for replacing single $\mathrm{S}$ with $\mathrm{O}$ atom. ${ }^{42}$

Next, we study the lateral diffusion of the $\mathrm{O}$ atom on the $\mathrm{MoS}_{2}$ surface. The minimum energy path and corresponding energy variation is shown in Fig. 1. Our analysis reveals that
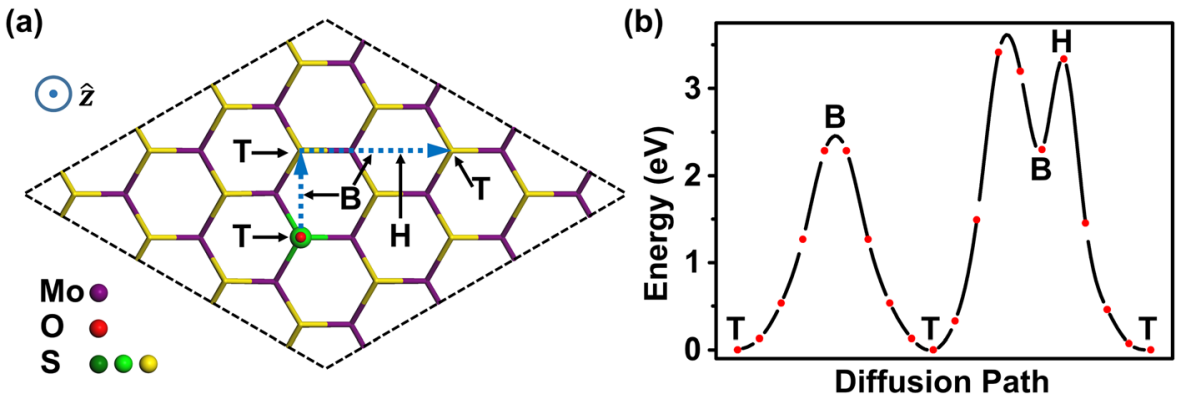

(c)

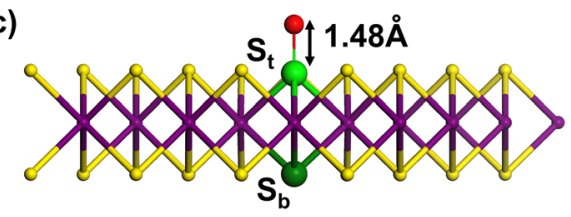

(d)

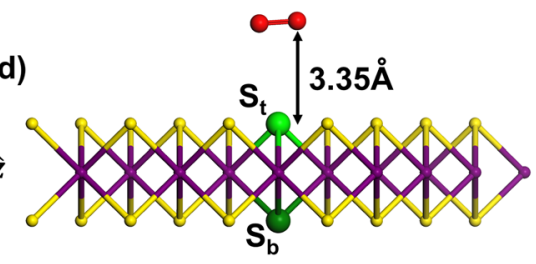

FIG. 1. (a) Top view of atomic oxygen adsorption. The adsorption cites are indicated by $\mathrm{T}$ (on top of $\mathrm{S}$ atom), B (in between two neighboring $\mathrm{S}$ atoms), and $\mathrm{H}$ (center of the hexagon). The lateral diffusion path is shown by dashed, blue arrows. (b) The energetics of oxygen lateral diffusion. Side view of (c) atomic and (d) molecular oxygen adsorption on $\mathrm{MoS}_{2}$ monolayer. z-axes is normal to the surface. Purple, red, and yellow spheres represent Mo, O, and $\mathrm{S}$ atoms, respectively. The $\mathrm{S}$ atoms interacting with oxygen is label as $S_{t}$ (top layer) and $\mathrm{S}_{b}$ (bottom layer) and are represented with light and dark green spheres, respectively. 
(a)
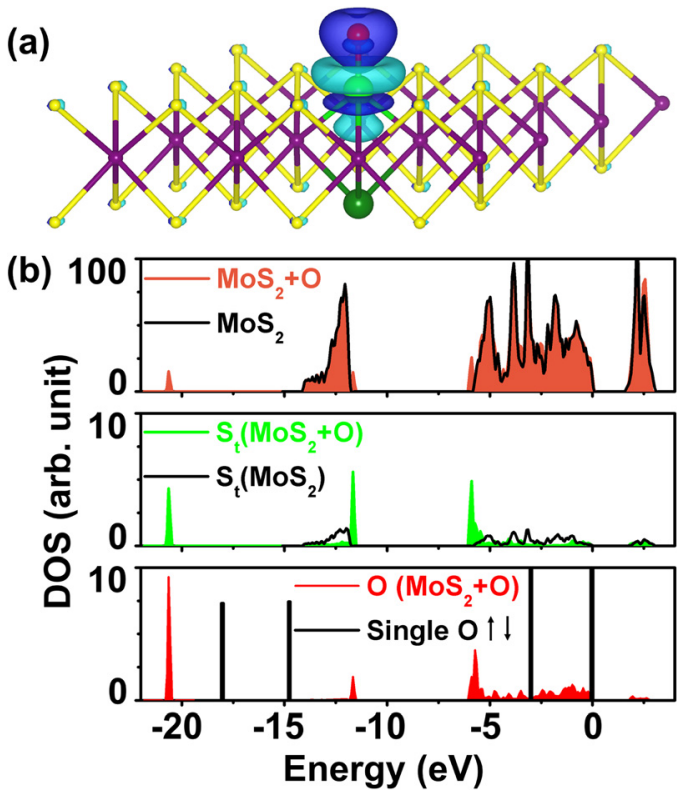

FIG. 2. (a) The difference charge density plot upon oxygen atom adsorption and (b) corresponding total density of states (top panel) and projected density of states on $\mathrm{S}_{t}$ (middle panel) and $\mathrm{O}$ (bottom panel) atoms before (solid black line) and after (coloured region) adsorption.

bridge (B) and hollow $(\mathrm{H})$ cites are metastable and the $\mathrm{O}$ atom stays only at the top $(\mathrm{T})$ site. The diffusion barrier for a single atomic $\mathrm{O}$ is high for all directions and calculated as $2.28 \mathrm{eV}$ for $\mathrm{T} \rightarrow \mathrm{B}, 3.41 \mathrm{eV}$ for $\mathrm{T} \rightarrow \mathrm{B} \rightarrow \mathrm{H}$, and 3.34 for $\mathrm{T} \rightarrow \mathrm{H}$.

In contrast to single $\mathrm{O}$ adsorption, the interaction of an $\mathrm{O}_{2}$ molecule with $\mathrm{MoS}_{2}$ is weak and it can only be physisorbed. Similar to the $\mathrm{O}$ atom case, we try various adsorption cites but we find that $\mathrm{O}_{2}$ does not chemically bind and stays at a physisorption distance of $\sim 3.40 \AA$ as shown in Fig. 1(c). Using Eq. (1), $E_{b}$ is calculated as $0.07 \mathrm{eV}$ when vdW corrections are included. The net magnetic moment of the system is $2 \mu_{B}$ indicating that $\mathrm{O}_{2}$ is still in the triplet state. Even if the initial position of $\mathrm{O}_{2}$ is chosen to be very close to $\mathrm{MoS}_{2}$ with elongated $\mathrm{O}-\mathrm{O}$ bond, the molecule $\mathrm{O}_{2}$ would rather move away from the surface than bind to it. Accordingly, ideal $\mathrm{MoS}_{2}$ does not dissociate $\mathrm{O}_{2}$ under normal conditions and dissociation requires a high external energy.

As a final step, we consider the interaction of another $\mathrm{O}$ atom with the adsorbate. Interestingly, when the incoming $\mathrm{O}$ atom approaches the adsorbed one on top, the $\mathrm{O}-\mathrm{S}_{t}$ bond is broken at a distance of $1.63 \AA$ and then $\mathrm{O}_{2}$ is formed. Afterwards, the $\mathrm{O}_{2}$ molecule moves away from the surface as expected. However, when the incoming $\mathrm{O}$ approaches from the side, it first interacts with $\mathrm{MoS}_{2}$ and binds to a nearby $\mathrm{S}$ atom on top. Even if these $\mathrm{O}$ atoms are forced to get in close proximity, they do not form an $\mathrm{O}_{2}$ molecule once they receive charge from $\mathrm{S}$ atoms. The configuration where two $\mathrm{O}$ atoms bind to neighboring $\mathrm{S}$ atom is energetically $1.1 \mathrm{eV}$ more favorable than the formation of $\mathrm{O}_{2}$ molecule.

In conclusion, our results indicate that even though $\mathrm{MoS}_{2}$ does not interact with $\mathrm{O}_{2}$ molecules, it can be easily oxidized by atomic oxygen. However, the lateral diffusion barrier is high for adsorbed atomic $\mathrm{O}$ and prevents its movement on the surface; a second $\mathrm{O}$ atom can break the O-S bond by forming $\mathrm{O}_{2}$ molecule.

\section{PENETRATION OF OXYGEN THROUGH $\mathrm{MOS}_{2}$ COATING}

\section{A. Ideal $\mathrm{MoS}_{2}$ monolayer}

Clarifying the interaction of oxygen with a $\mathrm{MoS}_{2}$ monolayer, we now address the vertical diffusion of atomic $\mathrm{O}$ and molecular $\mathrm{O}_{2}$ through $\mathrm{MoS}_{2}$. The minimum energy path and the resulting energy barriers of oxygen for vertical diffusion can reveal the possibility of using $\mathrm{MoS}_{2}$ as a protective layer. As we are not interested in a specific reactive surface, we mainly focus on suspended $\mathrm{MoS}_{2}$ monolayer. In this model, $\mathrm{MoS}_{2}$ is considered to be free-standing where only specific Mo atoms are fixed to prevent the displacement of the suspended layer and all other atoms are fully relaxed. This approach is expected to work for the cases where the surface- $\mathrm{MoS}_{2}$ interaction is not very strong and $\mathrm{MoS}_{2}$ has some flexibility to bend. For instance, Topsakal et al. ${ }^{15}$ calculated the diffusion barrier of oxygen through a graphene monolayer as 5.98 and $5.93 \mathrm{eV}$ with and without an underlaying $\mathrm{Al}$ (111) surface which indicates that the considered model yields realistic results.

We start with the penetration of atomic oxygen though suspended $\mathrm{MoS}_{2}$. Snapshots of the minimum energy path and corresponding energy variation are represented in Fig. 3(a). The path starts from the adsorption site where $\mathrm{O}$ atom is on top of $\mathrm{S}_{t}$. The $\mathrm{O}$ atom is then manually pushed vertically in the z-direction with $0.2 \AA$ increments. At each step, the lateral coordinates of $\mathrm{O}$ atom are relaxed but the perpendicular coordinate is kept fixed. $\mathrm{MoS}_{2}$ is considered to be free-standing where all atoms were fully relaxed except specific Mo atoms which were pinned to prevent displacement of the suspended layer. As can be noticed from the snapshots, the vertical movement of $\mathrm{O}$ atom pushes the $\mathrm{S}_{t}$ and $\mathrm{S}_{b}$ atoms and $\mathrm{MoS}_{2}$ is bent until $\mathrm{O}$ reaches the Mo-layer in the middle. The strong S-O interaction discussed in the previous section makes $\mathrm{O}$ penetration difficult yielding a high diffusion energy barrier $(\Delta \mathrm{E})$ which is calculated to be $13.94 \mathrm{eV}$. Notice that $\Delta \mathrm{E}$ is significantly larger than the reported barrier for suspended graphene $(5.98 \mathrm{eV}){ }^{15}$

When the surface- $\mathrm{MoS}_{2}$ interaction gets strong, the sulfur atoms at the bottom layer can bind covalently with the underlaying surface and are no longer free to move. In such cases, it is reasonable to fix the bottom sulfur layer of $\mathrm{MoS}_{2}$ while allowing other atoms to relax. Completely free and fixed bottom layer can model the two extreme cases and can set upper and lower boundaries for the oxygen diffusion barrier. When the bottom S-layer remains fixed, it prevents the $\mathrm{MoS}_{2}$ from bending during atomic $\mathrm{O}$ penetration. Therefore, the only possible diffusion path of the $\mathrm{O}$ atom is to replace $\mathrm{S}_{t}$ as shown in Fig. 3(b) and this significantly reduces $\Delta \mathrm{E}$ and calculated to be $4.88 \mathrm{eV}$. On the other hand, this time minimum energy path reveals a second energy barrier which emerges mainly due to the O-S $\mathrm{S}_{t}$ bond breaking during diffusion and it is calculated as $4.13 \mathrm{eV}$. Although $\Delta \mathrm{E}$ is reduced, two energy barriers still indicate the resistance of $\mathrm{MoS}_{2}$ 


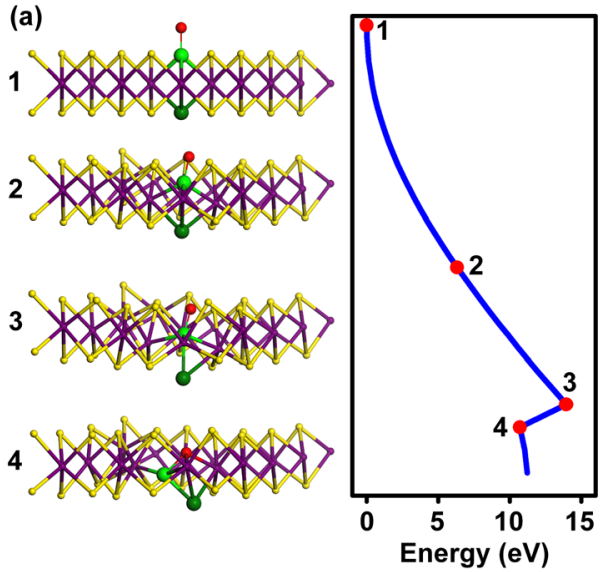

FIG. 3. The minimum energy path of an atomic oxygen penetration through an ideal $\mathrm{MoS}_{2}$ monolayer. (a) $\mathrm{MoS}_{2}$ is considered to be free-standing where only specific Mo atoms are fixed to prevent the displacement of the suspended layer and all other atoms are fully relaxed (b) The bottom sulfur layer of $\mathrm{MoS}_{2}$ is kept fixed while allowing other atoms to relax. against oxygen diffusion. We claim that this case results in a lower bound, because, although the substrate can prevent the bottom S-layer to move downwards to some extent, there will still be a bending of the $\mathrm{MoS}_{2}$ layer. Accordingly, $\Delta \mathrm{E}$ is expected to be in the range of $4.88-13.94 \mathrm{eV}$ as the considered models set lower and upper limits for $\Delta \mathrm{E}$.

Next, we consider vertical diffusion of $\mathrm{O}_{2}$ molecule keeping the same approach followed in the atomic $\mathrm{O}$ case. Snapshots of the minimum energy path and corresponding energy variation are represented in Fig. 4(a). The path starts with the adsorption site obtained in the previous section and then $\mathrm{O}_{2}$ molecule is forced to move vertically down by steps of $0.2 \AA$. Only the vertical coordinate of one of the $\mathrm{O}$ atoms is kept fixed and the other one is free to move. For the suspended $\mathrm{MoS}_{2}$ case, when $\mathrm{O}_{2}$ starts to approach $\mathrm{MoS}_{2}$ monolayer, it rotates and becomes perpendicular to the surface. Throughout the diffusion path, $\mathrm{O}_{2}$ pushes $\mathrm{S}_{t}$ and $\mathrm{S}_{b}$ until it expels them. $\Delta \mathrm{E}$ is high and is calculated to be $11.69 \mathrm{eV}$. The total energy of the system reduces when $S_{b}$ is expelled and $\mathrm{S}_{t}$ substitutes it and the vacant $\mathrm{S}_{t}$ is filled by oxygen.

As discussed in the atomic $\mathrm{O}$ case, free-standing $\mathrm{MoS}_{2}$ is an extreme case and thus $S_{b}$ may not be pulled of from the monolayer when there is an underlying surface (Fig. 4(b)). To include this effect, once again we fix the bottom sulfur layer of $\mathrm{MoS}_{2}$ while allowing other atoms to relax. In this path, once again the $\mathrm{O}_{2}$ molecule approaches the surface in the vertical direction and pushes $S_{t}$ but this time $S_{b}$ is pinned, and the $\mathrm{O}_{2}$ molecule dissociates and one of the $\mathrm{O}$ atom replaces $\mathrm{S}_{t}$ while the remaining one forms a new bond with $\mathrm{S}_{t}$. This diffusion path gives rise to three energy barriers which are calculated to be 3.94, 1.98, and 3.63 as shown in Fig. 4(b).

Our results indicate that the calculated $\Delta \mathrm{E}$ is high enough to prevent the penetration of oxygen atom/molecule through ideal $\mathrm{MoS}_{2}$. The strong and directional bonding between $\mathrm{O}$ and $\mathrm{S}$ atoms makes the vertical diffusion very difficult. Oxygen cannot penetrate though $\mathrm{MoS}_{2}$ without expelling or replacing the $\mathrm{S}$ atom(s) and these both require high energies. $\Delta \mathrm{E}$ depends on the $\mathrm{MoS}_{2}$-surface interaction and is higher when $\mathrm{MoS}_{2}$ is free to bend and is therefore maximum for the suspended case.

\section{B. $\mathrm{MoS}_{2}$ monolayer with defects}

The above analysis was limited to continuous and defect-free $\mathrm{MoS}_{2}$. In the literature, various type of defects in $\mathrm{MoS}_{2}$ have been reported ${ }^{43,44}$ and thus a $\mathrm{MoS}_{2}$ coating may contain vacancies in practice. Among possible defects, we consider three vacancy types, namely single sulfur $\left(\mathrm{S}_{v}\right)$, single Mo $\left(\mathrm{Mo}_{v}\right)$, and single Mo and two sulfur $\left((\mathrm{Mo}+2 \mathrm{~S})_{v}\right)$ atom(s) vacancies as shown in Fig. 5(a) and we examine O/ $\mathrm{O}_{2}$ adsorption and vertical diffusion. The obtained results are summarized in Table I. Introducing a vacancy changes the adsorption profile and the corresponding structures upon $\mathrm{O}$ and $\mathrm{O}_{2}$ adsorption are shown in Figs. 5(b) and 5(c). When there is a S-vacancy in the monolayer $\left(\mathrm{S}_{v}\right.$ or $\left.(\mathrm{Mo}+2 \mathrm{~S})_{v}\right)$, the $\mathrm{O}$ atom fills the vacancy, substituting the missing $\mathrm{S}$ and (a)

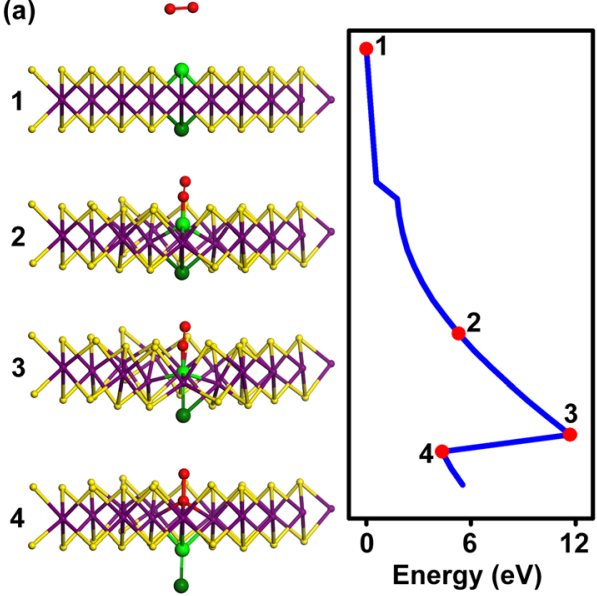

(b)

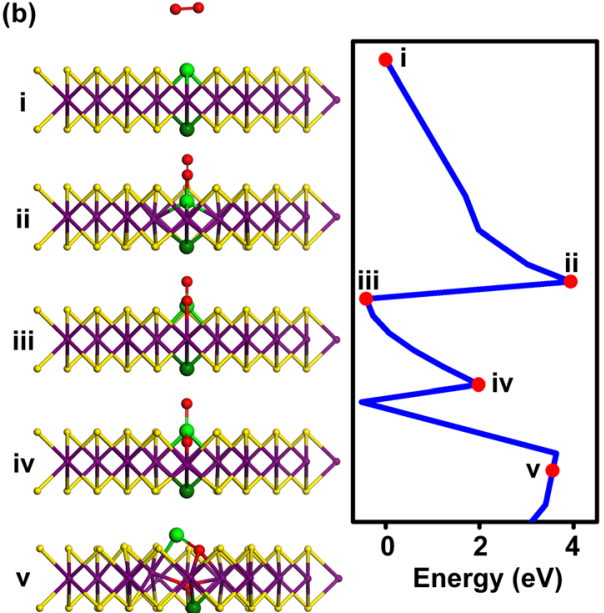

FIG. 4. The minimum energy path of oxygen molecule penetration through an ideal $\mathrm{MoS}_{2}$ monolayer. (a) $\mathrm{MoS}_{2}$ is considered to be free-standing where only specific Mo atoms are fixed to prevent the displacement of the suspended layer and all other atoms are fully relaxed. (b) The bottom sulfur layer of $\mathrm{MoS}_{2}$ is kept fixed while allowing other atoms to relax. 


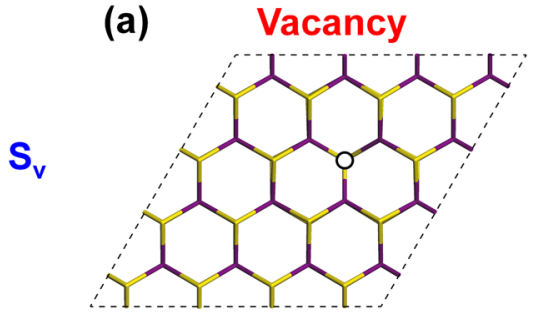

(b)

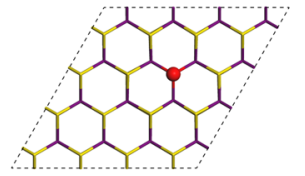

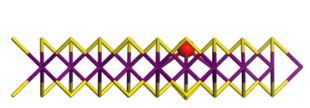

$\mathrm{Mo}_{\mathrm{v}}$

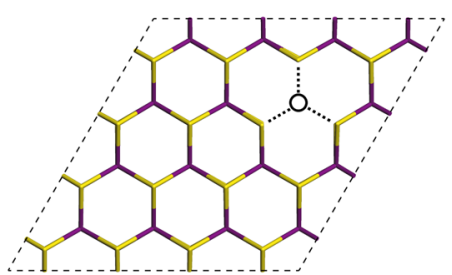

(c) $\mathrm{O}_{2}$
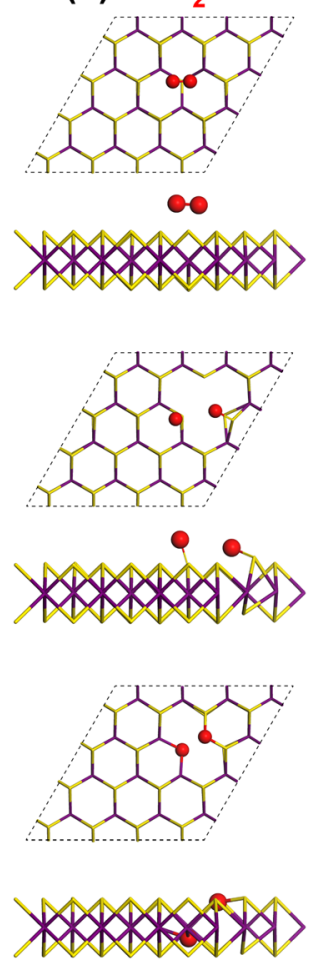

FIG. 5. The structure of $\mathrm{MoS}_{2}$ with (a) single sulfur $\left(\mathrm{S}_{v}\right)$, single $\mathrm{Mo}\left(\mathrm{Mo}_{v}\right)$, and single $\mathrm{Mo}$ and two sulfur $(\mathrm{Mo}+2 \mathrm{~S})_{v}$ vacancies. (b) Side and top view of oxygen atom and (c) oxygen molecule adsorption on defected $\mathrm{MoS}_{2}$. binds to Mo. ${ }^{31}$ Using Eq. (1) (considering the total energy of defected $\left.\mathrm{MoS}_{2}\right), \mathrm{E}_{b}$ is calculated as 7.40 and $7.76 \mathrm{eV}$ for $\mathrm{S}_{v}$ and $(\mathrm{Mo}+2 \mathrm{~S})_{v}$, respectively. In the case of $\mathrm{Mo}_{v}$, the $\mathrm{O}$ atom binds to $\mathrm{S}$ atom with $\mathrm{E}_{b}$ of $4.07 \mathrm{eV}$ which is almost equal to the ideal $\mathrm{MoS}_{2}$ case. The $\mathrm{O}_{2}$ molecule weakly interacts with $\mathrm{MoS}_{2}$ when there is $\mathrm{S}_{v}$ yielding only $0.12 \mathrm{eV}$ binding energy when vdW correction is included. When there is $\mathrm{Mo}_{v}$ or $(\mathrm{Mo}+2 \mathrm{~S})_{v}, \mathrm{O}_{2}$ molecule still weakly interacts with $\mathrm{MoS}_{2}$. However, this state is metastable and $\mathrm{O}_{2}$ can easily dissociate once overcoming a small energy barrier and then $\mathrm{O}$ atoms bind to $S_{t}$ atoms with a high binding energy.

For each type of vacancy, we examine the vertical diffusion of atomic and molecular oxygen separately following the same methodology. The path starts from the adsorption geometry as shown in Figs. 5(b) and $5(\mathrm{c})$ then $\mathrm{O} / \mathrm{O}_{2}$ is pushed vertically with $0.2 \AA$ increments while relaxing the system at each step except for the z-coordinate of oxygen. The minimum energy path and the corresponding energy barriers are illustrated in Fig. 6. Our analysis indicates that

TABLE I. The binding energy $\mathrm{E}_{b}$, the binding energy $\mathrm{E}_{b}^{V d W}$ with van der Waals correction, and vertical diffusion energy barrier $\Delta E$ are reported for $\mathrm{O}$ atom and $\mathrm{O}_{2}$ molecule on defected and defect-free $\mathrm{MoS}_{2}$.

\begin{tabular}{lcccc}
\hline \hline & Vacany & $E_{b}(\mathrm{eV})$ & $E_{b}^{V d W}(\mathrm{eV})$ & $\Delta E(\mathrm{eV})$ \\
\hline O atom & $\ldots$ & 3.93 & 4.07 & $4.88-13.94$ \\
& $\mathrm{~S}$ & 7.24 & 7.40 & 7.40 \\
& $\mathrm{Mo}$ & 3.89 & 4.02 & $0.13,0.40$ \\
& $\mathrm{Mo}+2 \mathrm{~S}$ & 7.61 & 7.76 & 4.00 \\
$\mathrm{O}_{2}$ molecule & $\ldots$ & 0.00 & 0.07 & $3.94-11.69$ \\
& $\mathrm{~S}$ & 0.00 & 0.12 & 4.32 \\
& $\mathrm{Mo}$ & 1.70 & 1.92 & $0.23,0.38$ \\
& $\mathrm{Mo}+2 \mathrm{~S}$ & 5.16 & 5.51 & 2.49 \\
\hline \hline
\end{tabular}

$\Delta$ E significantly decreases when $\mathrm{MoS}_{2}$ contains defects. The effect is less significant for $\mathrm{S}_{v}$ where $\Delta \mathrm{E}$ becomes 7.80 and $4.32 \mathrm{eV}$ for $\mathrm{O}$ and $\mathrm{O}_{2}$, respectively. For the other types of vacancy, $\Delta \mathrm{E}$ drastically reduces and is calculated to be even less than $0.5 \mathrm{eV}$ for $\mathrm{Mo}_{v}$. To the best of our knowledge, $\mathrm{Mo}_{v}$ is not a common defect type and has yet not been found experimentally. We conclude that vacant formation weakens oxidation protection of the $\mathrm{MoS}_{2}$ coating but still blocks oxygen diffusion to some extend. Therefore, multiple layers of $\mathrm{MoS}_{2}$ coating should be considered for efficient protection.

\section{Alternative TMD structures: Monolayer of $\mathbf{W S}_{\mathbf{2}}$}

As our results indicate that strong $\mathrm{S}-\mathrm{O}$ interaction is a critical parameter to determine the oxidation resistance, we consider $\mathrm{WS}_{2}$, whose bulk form is used in macro scale coating, as an alternative material in the class of TMDs. Twodimensional $\mathrm{WS}_{2}$ has also been synthesized and its novel properties has been revealed. ${ }^{16,45} \mathrm{WS}_{2}$ has a similar crystal structure as $\mathrm{MoS}_{2}$ and is composed of vertically stacked layers with an interlayer distance of $6.24 \AA$. We start with the oxidation of $\mathrm{WS}_{2}$ and obtain similar results as found for $\mathrm{MoS}_{2}$. While the $\mathrm{O}$ atom is chemisorbed on $\mathrm{WS}_{2}$ forming a strong bond with $\mathrm{S}\left(E_{b}=3.95 \mathrm{eV}, d_{S-O}=1.48 \AA\right), \mathrm{O}_{2}$ molecule weakly interacts with $\mathrm{WS}_{2}\left(E_{b}=0.05 \mathrm{eV}, d_{S-O}>3 \AA\right)$. Fig. 1 roughly represents the optimized structures.

The penetration of $\mathrm{O} / \mathrm{O}_{2}$ is studied using similar methodology and minimum energy paths are found to resemble those for the case of $\mathrm{MoS}_{2}$. For ideal, suspended $\mathrm{WS}_{2}, \Delta \mathrm{E}$ is calculated as 9.26 and $7.26 \mathrm{eV}$ for $\mathrm{O}$ atom and $\mathrm{O}_{2}$ molecule, respectively. Note that $\Delta \mathrm{E}$ is smaller than those obtained for ideal $\mathrm{MoS}_{2}$. For $\mathrm{WS}_{2}$, however, the S-O interaction is very strong, it is slightly weaker than the one for $\mathrm{MoS}_{2}$ and this can explain the reduction in the diffusion barrier. $\Delta \mathrm{E}$ is still 


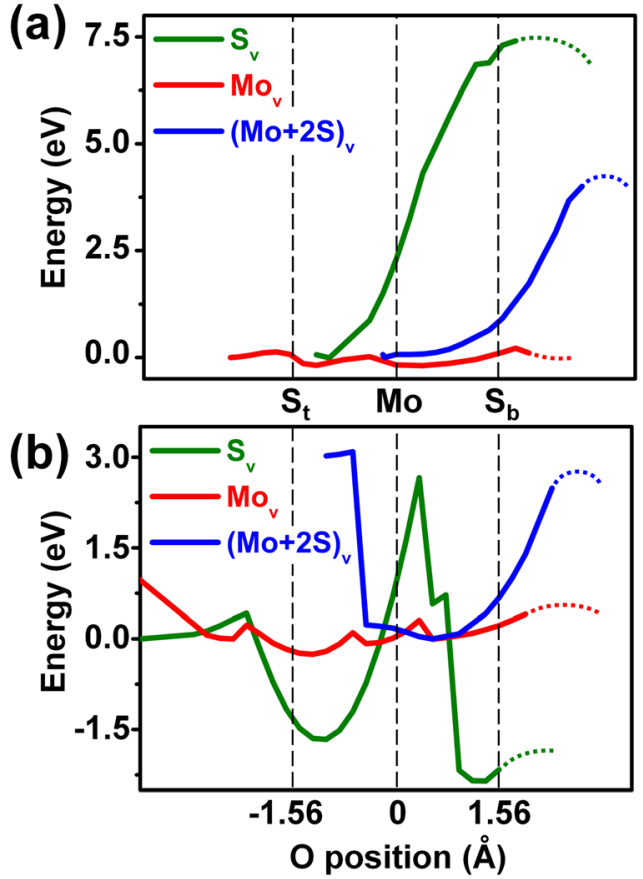

FIG. 6. The minimum energy path of (a) atomic and (b) molecular oxygen vertical diffusion through $\mathrm{MoS}_{2}$ with defects. The vertical dashed lines indicate the position of the Mo- and S-layers in $\mathrm{MoS}_{2}$.

very high to prevent penetration of atomic and molecular oxygen and indicates that $\mathrm{WS}_{2}$, and similar systems in the same class can also be considered as protective nanocoating material.

\section{CONCLUSIONS}

In conclusion, we showed that (1) while $\mathrm{MoS}_{2}$ weakly interacts with molecular oxygen, it can be easily oxidized by oxygen atoms, (2) continuous and defect-free $\mathrm{MoS}_{2}$ can serve as an ideal nanocoating material which can protect the underlying surface from oxidation. The strong sulfur-oxygen interaction makes penetration of oxygen atom/molecule through the monolayer very difficult which gives rise to high energy barriers, and (3) the vertical diffusion barrier reduces when vacancies are introduced but is still high enough for most of the cases to resist against oxygen penetration. A similar trend is obtained for $\mathrm{WS}_{2}$ which indicates that the results can be generalized for the systems in the same class.

\section{ACKNOWLEDGMENTS}

This work was supported by the bilateral project between TUBITAK (through Grant No. 113T050) and Flemish Science Foundation (FWO-Vl). The calculations were performed at TUBITAK ULAKBIM, High Performance and Grid Computing Center (TR-Grid eInfrastructure). E.D. acknowledges support from Bilim Akademisi-The Science Academy, Turkey under the BAGEP program. H.S. is supported by an FWO Pegasuslong Marie Curie Fellowship.

${ }^{1}$ D. M. Merkula, P. D. Novikov, V. N. Ivanenkov, V. V. Sapozhnikov, and Y. I. Lyakhin, Oceanology 14, 299300 (1974).

${ }^{2}$ M. I. Redondo and C. B. Breslin, Corros. Sci. 49, 1765-1776 (2007).
${ }^{3}$ J. E. Gray and B. Luan, J. Alloy Compd. 336, 88-113 (2002).

${ }^{4}$ B. V. A. Rao, M. Y. Iqbal, and B. Sreedhar, Corros. Sci. 51, 1441-1452 (2009).

${ }^{5}$ M. Stratmann, R. Feser, and A. Leng, Electrochim. Acta. 39, 1207-1214 (1994).

${ }^{6}$ M. Segarra, L. Miralles, J. Diaz, H. Xuriguera, J. M. Chimenos, F. Espiell, and S. Pinol, Mater. Sci. Forum 426-432, 3511-3516 (2003).

${ }^{7}$ K. Novoselov, A. K. Geim, S. Morozov, D. Jiang, M. Katsnelson, I. Grigorieva, S. Dubonos, and A. Firsov, Nature 438, 197-200 (2005).

${ }^{8}$ Y. S. Dedkov, M. Fonin, U. Rudiger, and C. Laubschat, Appl. Phys. Lett. 93, 022509 (2008).

${ }^{9}$ Y. S. Dedkov, M. Fonin, and C. Laubschat, Appl. Phys. Lett. 92, 052506 (2008).

${ }^{10}$ B. Borca, F. Calleja, J. J. Hinarejos, A. L. V. Parga, and R. Miranda, J. Phys.: Condens. Matter. 21, 134002 (2009).

${ }^{11}$ S. Chen, L. Brown, M. Levendorf, W. Cai, S. Y. Ju, J. Edgeworth, X. Li, C. W. Magnuson, A. Velamakanni, R. D. Piner, J. Kang, J. Park, and R. S. Ruoff, ACS Nano. 5, 1321-1327 (2011).

${ }^{12}$ S. Gadipelli, I. Calizo, J. Ford, G. Cheng, A. R. H. Walker, and T. Yildirim, J. Mater. Chem. 21, 16057-16065 (2011).

${ }^{13}$ J. Cho, L. Gao, J. Tian, H. Cao, W. Wu, Q. Yu, E. N. Yitamben, B. Fisher, J. R. Guest, Y. P. Chen, and N. P. Guisinger, ACS Nano. 5, 3607-3613 (2011).

${ }^{14}$ N. A. Vinogradov, K. Schulte, M. L. Ng, A. Mikkelsen, E. Lundgren, N. Martensson, and A. B. Preobrajenski, J. Phys. Chem. C. 115, 9568-9577 (2011).

${ }^{15}$ M. Topsakal, H. Sahin, and S. Ciraci, Phys. Rev. B. 85, 155445 (2012).

${ }^{16}$ J. N. Coleman, M. Lotya, A. O’Neill, S. D. Bergin, P. J. King, U. Khan, K. Young, A. Gaucher, S. De, R. J. Smith, I. V. Shvets, S. K. Arora, G. Stanton, H.-Y. Kim, K. Lee, G. T. Kim, G. S. Duesberg, T. Hallam, J. J. Boland, J. J. Wang, J. F. Donegan, J. C. Grunlan, G. Moriarty, A. Shmeliov, R. J. Nicholls, J. M. Perkins, E. M. Grieveson, K. Theuwissen, D. W. McComb, P. D. Nellist, and V. Nicolosi, Science 331, 568-571 (2011).

${ }^{17}$ M. Chhowalla and G. A. J. Amaratunga, Nature 407, 164-167 (2000).

${ }^{18}$ C. Donnet, J. M. Martin, Th. Le Mogne, and M. Belin, Tribol. Int. 29, 123-128 (1996).

${ }^{19}$ H. H. Chien, K. J. Ma, S. V. P. Vattikuti, C. H. Kuo, C. B. Huo, and C. L. Chao, Thin Solid Films. 518, 7532-7534 (2010).

${ }^{20}$ K. S. Novoselov, D. Jiang, T. Booth, V. V. Khotkevich, S. M. Morozov, and A. K. Geim, Proc. Natl. Acad. Sci. U.S.A. 102, 10451-10453 (2005).

${ }^{21}$ K. K. Kam and B. A. Parkinson, J. Phys. Chem. 86, 463-467 (1982).

${ }^{22}$ K. F. Mak, C. Lee, J. Hone, J. Shan, and T. F. Heinz, Phys. Rev. Lett. 105, 136805 (2010).

${ }^{23}$ C. Ataca, H. Sahin, and S. Ciraci, J. Phys. Chem. C 116, 8983-8999 (2012).

${ }^{24}$ J. P. Wilcoxon, T. R. Thurston, and J. E. Martin, Nanostruct. Mater. 12, 993-997 (1999).

${ }^{25}$ B. Abrams and J. Wilcoxon, Crit. Rev. Solid State Mater. Sci. 30, 153-182 (2005).

${ }^{26}$ B. Radisavljevic, A. Radenovic, J. Brivio, V. Giacometti, and A. Kis, Nat. Nanotechnol. 6, 147-150 (2011).

${ }^{27}$ W. Park, J. Park, J. Jang, H. Lee, H. Jeong, K. Cho, S. Hong, and T. Lee, Nanotechnology 24, 095202 (2013).

${ }^{28}$ G. Kline, K. K. Kam, R. Ziegler, and B. A. Parkinson, Sol. Energy Mater. 6, 337-350 (1982).

${ }^{29}$ E. Gourmelon, O. Lignier, H. Hadouda, G. Couturier, J. C. Bernède, J. Tedd, J. Pouzet, and J. Salardenne, Sol. Energy Mater Sol. Cells 46, 115-121 (1997)

${ }^{30}$ J. He, K. Wu, R. Sa, Q. Li, and Y. Wei, Appl. Phys. Lett. 96, 082504 (2010).

${ }^{31}$ A. Azcatl, S. McDonnell, K. C. Santosh, X. Peng, H. Dong, X. Qin, R. Addou et al., Appl. Phys. Lett. 104, 111601 (2014).

${ }^{32}$ W. Kohn and L. J. Sham, Phys. Rev. 140, A1133-A1138 (1965).

${ }^{33}$ P. Hohenberg and W. Kohn, Phys. Rev. 136, B864-B871 (1964).

${ }^{34}$ G. Kresse and J. Hafner, Phys. Rev. B. 47, 558-561 (1993).

${ }^{35}$ G. Kresse and J. Furthmuller, Phys. Rev. B. 54, 11169-11186 (1996).

${ }^{36}$ J. P. Perdew, K. Burke, and M. Ernzerhof, Phys. Rev. Lett. 77, 3865-3868 (1996).

${ }^{37}$ P. E. Blochl, Phys. Rev. B. 50, 17953-17979 (1994).

${ }^{38}$ H. J. Monkhorst and J. D. Pack, Phys. Rev. B. 13, 5188-5192 (1976).

${ }^{39}$ G. H. Diercksen and S. Wilson, Methods in Computational Molecular Physics (Springer, 1983).

${ }^{40}$ G. Mills, H. Jonsson, and G. K. Schenter, Surf. Sci. 324, 305-337 (1995). 
${ }^{41}$ G. Henkelman, A. Arnaldsson, and H. Jonsson, Comput. Mater. Sci. 36, 354-360 (2006).

${ }^{42}$ On the other hand, even energetically not favourable when compared to the ideal $\mathrm{MoS}_{2}$, the resulting structure $\left(\mathrm{Mo}_{16} \mathrm{~S}_{15} \mathrm{O}_{1}\right)$ can indicate the possibility of formation of stable molybdenum oxide $\left(\mathrm{MoO}_{x}\right)$ by convenient processes.
${ }^{43}$ W. Zhou, X. Zou, S. Najmaei, Z. Liu, Y. Shi, J. Kong, J. Lou, P. M. Ajayan, B. I. Yakobson, and J.-C. Idrobo, Nano Lett. 13, 2615-2622 (2013).

${ }^{44}$ S. McDonnell, R. Addou, C. Buie, R. M. Wallace, and C. L. Hinkle, ACS Nano 8, 2880-2888 (2014).

${ }^{45}$ B. H. Xu, B. Z. Lin, D. Y. Sun, C. Ding, X.-Z. Liu, and Z.-J. Xiao, Mater, Res. Bull. 42, 1633-1639 (2007). 